\title{
Nutritional assessment and surgical outcomes in very elderly patients undergoing pancreaticoduodenectomy: a retrospective study
}

Masashi Utsumi ( $\square$ masashi11232001@yahoo.co.jp )

Department of Surgery, National Hospital Organization, Iwakuni Clinical Center

Hideki Aoki

Iwakni Clinical Center

Seichi Nagahisa

Iwakuni Clinical Center

Yuta Une

Iwakuni Clinical Center

Yuji Kimura

Iwakuni Clinical Center

Megumi Watanabe

Iwakuni Clincal Center

Fumitaka Taniguchi

Iwakuni Clincal Center

Takashi Arata

Iwakuni Clincal Center

Koh Katsuda

Iwakuni Clincal Center

Kohji Tanakaya

Iwakuni Clincal Center

Research

Keywords: pancreaticoduodenectomy, elderly patients, nutritional factors

Posted Date: July 8th, 2020

DOI: https://doi.org/10.21203/rs.3.rs-40426/v1

License: (1) (1) This work is licensed under a Creative Commons Attribution 4.0 International License.

Read Full License 
Version of Record: A version of this preprint was published at Surgery Today on October 26th, 2020. See the published version at https://doi.org/10.1007/s00595-020-02169-2. 


\section{Abstract}

Background Conflicting data on the safety of pancreaticoduodenectomy (PD) for elderly patients exist. Therefore, this study aimed to evaluate and compare the nutritional factors and clinical outcomes of PD between elderly and non-elderly patients.

Methods A retrospective study of 122 consecutive patients who underwent PD from April 2008 to April 2020 was conducted. Preoperative and postoperative nutritional factors (prognostic nutritional index [PNI]), complication rates, and survival rates were compared between the elderly (age $\geq 80$ years) and non-elderly (age $<80$ years) patient groups. Furthermore, changes in nutrition markers were evaluated before surgery to 1 year after surgery. Data were analyzed using unpaired Student's $t$-test, chi-squared test with Fisher's exact test, and log-rank test.

Results A total of 20 elderly patients (16.4\%) and 102 non-elderly patients (83.6\%) underwent PD. With respect to preoperative factors, elderly patients had a significantly lower PNI than non-elderly patients. The duration of operation, amount of blood loss, postoperative complication rate, and incidence rate of pancreatic fistula were similar between the two groups. At 3 months postoperatively, elderly patients had a lower albumin level and PNI than non-elderly patients. The median length of hospital stay was significantly longer in the elderly group than in the non-elderly group ( 39.9 vs. 27 days, $P=0.004$ ). The rate of death due to other diseases was relatively higher in the elderly group than in the non-elderly group. Elderly patients had a significantly lower overall survival rate than non-elderly patients (1-/3-/5-year overall survival rates: $78.1 \% / 26.7 \% / 13.3 \%$ vs. $87.1 \% / 54.4 \% / 46.7 \%$; log-rank test, $P=0.008$ ).

Conclusions Elderly patients had a lower nutritional status and lower survival rate than non-elderly patients. Careful patient selection and optimal perioperative care are necessary to determine whether PD is indicated for elderly patients.

\section{Background}

Pancreaticoduodenectomy (PD) offers a chance for cure in patients with pancreatic cancer involving the head of the pancreas and other periampullary malignancies [1, 2]. Furthermore, PD is a valid treatment option for selected patients with benign tumors and non-cancerous conditions, including chronic pancreatitis. The overall morbidity and mortality associated with PD range from $45 \%$ to $52 \%[3,4]$ and from $0 \%$ to $5 \%[5-8]$, respectively.

The population of individuals aged $>80$ years is increasing globally, accounting for approximately $1 \%$ of the total population, and it is predicted to increase four-fold by 2050 [9]. There will be an increasing need to consider patients older than 80 years of age for PD, particularly for the treatment of pancreatic cancer, for which the incidence increases with age [9].

The outcomes of PD have improved with better surgical techniques and postoperative care of patients [9]. However, performing this surgery in elderly patients is still a controversial issue. In many studies about 
PD, the elderly cohort accounted for a very small proportion of the study population. There may be selection bias for the indication of PD. Compared to non-elderly patients, elderly patients are usually frail and malnourished and have more common diseases.

The morbidity and mortality after PD are associated with malnutrition. Nutritional support may reduce postoperative complications in patients undergoing PD [10]. A low preoperative albumin level has been associated with an increased risk of morbidity and mortality in patients undergoing PD [11]. The prognostic nutritional index (PNI) and platelet-to-lymphocyte ratio are useful prognostic indicators for pancreatic cancer patients after PD [12]. However, the association between elderly age and nutrition status is unclear during the perioperative period. There exist few reports on postoperative changes in nutritional indexes in elderly patients.

This study aimed to evaluate and compare the nutritional factors and clinical outcomes of PD between elderly (age $\geq 80$ years) and non-elderly (age $<80$ years) patients as well as to examine the effect of PD on changes in nutritional indexes to monitor patients during postoperative follow-up. As appropriate preoperative evaluation of elderly patients will lead to their safe management, we investigated nutritional parameters, including the albumin level, hemoglobin level, platelet count, lymphocyte count, and PNI, before surgery to 1 year after PD [13].

\section{Methods}

\section{Ethical statements}

Patients were not required to provide informed consent for the study because the analysis used anonymous data that were obtained after patients agreed to treatment by written consent. The ethics committee of the Iwakuni Clinical Center approved the study protocol (approval number: 0138). This study was performed in accordance with the protocols of the 1975 Declaration of Helsinki.

\section{Patients, data collections, and definitions}

This retrospective study included 122 consecutive patients who underwent PD in the Department of Surgery of the Iwakuni Clinical Center from April 2008 to April 2020.

We compared preoperative and postoperative nutritional factors, $\mathrm{PNI}$, complication rates, and survival rates between the elderly (age $\geq 80$ years) and non-elderly (age $<80$ years) patient groups. Preoperative blood samples were obtained within 2 weeks before surgery. The following preoperative patient data were collected: sex, age, hypertension, diabetes, alcohol consumption, smoking, body mass index (BMI), use of anticoagulants, indication for surgery, main pancreatic duct (MPD) diameter by preoperative computed tomography or magnetic resonance cholangiopancreatography, blood transfusion, blood loss, operative time, pancreaticojejunostomy technique, and tumor stage as per the Union for International Cancer Control (UICC) classification [14]. 
As for nutritional assessment, perioperative time-dependent changes in the nutrition markers, including the serum albumin level, hemoglobin level, lymphocyte count, and PNI score, were evaluated from before surgery to 1 year after surgery (at 1 week, 1 month, 3 months, and 1 year postoperatively) and compared between the two groups. PNI was calculated according to the following formula: 10xserum albumin level $(\mathrm{g} / \mathrm{dL})+0.05 \times$ total lymphocyte count $\left(\right.$ per $\left.\mathrm{mm}^{3}\right)$.

Specimens were histopathologically identified as and categorized into either malignant or benign tumor. A microscopic positive margin (R1) was defined as the presence of a tumor at the surgical margin at the time of histological examination.

Postoperative complications were graded according to the Clavien-Dindo classification [10], which was validated in pancreatic surgery. Grade 3 or 4 complications were considered as major. Pancreatic fistula was diagnosed and graded in accordance with the International Study Group on Pancreatic Fistula classification $[15,16]$. Perioperative mortality was defined as death within 30 days from operation or during hospitalization.

\section{Operative procedure}

Patients underwent subtotal stomach-preserving PD, which was performed by two surgeons who specialized in pancreatic surgery. All operations were performed via an open approach, and the degree of locoregional lymphadenectomy was determined according to the patient's preoperative diagnosis. Surgical reconstruction was performed using a modification of the Child method [17]. The proximal jejunal stump was passed through the retrocolic pathway, and pancreaticojejunostomy, biliojejunostomy, and gastrojejunostomy were subsequently performed. Pancreaticojejunostomy was performed using the modified Kakita anastomosis ( $n=47$; April 2008 to May 2013) or the modified Blumgart anastomosis ( $n=75$; June 2013 to present) [18]. During the procedure, the surgeon decided whether plastic stents for internal drainage needed to be inserted into the MPD. Two or three abdominal drains were placed anteriorly or posteriorly to the pancreaticojejunostomy anastomosis and hepaticojejunostomy anastomosis.

\section{Statistical analysis}

Continuous data are expressed as mean \pm standard deviation. Statistical analyses were conducted using unpaired Student's $t$-test and chi-squared test with Fisher's exact test. Overall patient survival was evaluated using the Kaplan-Meier method and compared with the log-rank test. A $P$-value $<0.05$ was considered statistically significant. Statistical analysis was performed using JMP software version 9 (SAS Institute, Cary, NC, USA).

\section{Results}

\section{Patients' characteristics}


Patients' clinicopathologic characteristics according to age are summarized in Table 1. Of the 122 patients who underwent PD, 20 (16.4\%) were 80 years or older of age. The elderly group had more poorrisk patients with respect to the American Society of Anesthesiologists (ASA) score $(P=0.002)$. Elderly patients were significantly more likely to have cardiac disease including coronary artery disease and arrhythmia than non-elderly patients $(P=0.038)$. No significant differences in sex, $\mathrm{BMI}$, drinking history, smoking history, and use of anticoagulants were found between the two groups. With regard to pathologic results, all elderly patients undergoing PD had malignancy, whereas $78.4 \%$ of non-elderly patients had malignancy $(P=0.003)$. Furthermore, more elderly patients underwent R1 resection than nonelderly patients $(P=0.027)$.

Table 1 Clinicopathological characteristics of patients undergoing pancreaticoduodenectomy by age group

\begin{tabular}{|c|c|c|c|}
\hline & $\begin{array}{l}\text { Elderly group } \\
(n=20)\end{array}$ & $\begin{array}{c}\text { Non-elderly group } \\
(n=102)\end{array}$ & $\begin{array}{c}P \text { - } \\
\text { value }\end{array}$ \\
\hline Mean age (years) & $83.1 \pm 2.9$ & $66.8 \pm 9.7$ & $<0.001$ \\
\hline Sex (male/female) & $11 / 9$ & $45 / 57$ & 0.942 \\
\hline BMI $\left(\mathrm{kg} / \mathrm{m}^{2}\right)$ & $19.8 \pm 3.1$ & $19.8 \pm 2.9$ & 0.982 \\
\hline ASA physical status grading system & & & 0.002 \\
\hline I & 0 & 11 & \\
\hline II & 11 & 78 & \\
\hline III & 9 & 13 & \\
\hline \multicolumn{4}{|l|}{ Comorbidity } \\
\hline Hypertension & 11 & 34 & 0.173 \\
\hline Diabetes mellitus & 4 & 27 & 0.535 \\
\hline Cardiac disease & 5 & 9 & 0.038 \\
\hline Cerebral disease & 2 & 10 & 0.978 \\
\hline Indication for surgery & & & 0.013 \\
\hline Pancreatic cancer & 7 & 41 & \\
\hline IPMN & 0 & 16 & \\
\hline Bile duct cancer & 9 & 16 & \\
\hline Ampullary cancer & 3 & 10 & \\
\hline Duodenal cancer & 0 & 5 & \\
\hline Chronic pancreatitis & 0 & 5 & \\
\hline Gallbladder cancer & 1 & 3 & \\
\hline Others (AIP, NET, metastasis, and SCN) & 0 & 6 & \\
\hline Malignant disease & $20(100)$ & $80(78.4)$ & 0.003 \\
\hline Drinking history & $7(35)$ & $50(49)$ & 0.465 \\
\hline Smoking history & $9(45)$ & $55(53.9)$ & 0.889 \\
\hline Use of anticoagulants & 3 (15) & $11(10.8)$ & 0.589 \\
\hline $\begin{array}{l}\text { MPD diameter on preoperative CT or MRCP } \\
(\mathrm{mm})\end{array}$ & $4.2 \pm 2.8$ & $3.7 \pm 1.9$ & 0.326 \\
\hline \multicolumn{4}{|l|}{ Preoperative laboratory data } \\
\hline Hemoglobin level (g/L) & $11.1 \pm 1.7$ & $12.5 \pm 1.6$ & $<0.001$ \\
\hline Platelet count $\left(\times 10^{4} / \mathrm{mm}^{3}\right)$ & $21.2 \pm 1.6$ & $22.9 \pm 0.7$ & 0.327 \\
\hline Total neutrophil count $\left(/ \mathrm{mm}^{3}\right)$ & $3976 \pm 401$ & $3492 \pm 178$ & 0.272 \\
\hline Total lymphocyte count $\left(/ \mathrm{mm}^{3}\right)$ & $1165 \pm 391$ & $1427 \pm 517$ & 0.034 \\
\hline Serum albumin level (g/dL) & $3.3 \pm 0.6$ & $3.9 \pm 0.5$ & $<0.001$ \\
\hline & $1.52 \pm 2.31$ & $1.18 \pm 2.77$ & 0.601 \\
\hline \multicolumn{4}{|l|}{ Nutrition marker } \\
\hline PNI & $39.2 \pm 6.9$ & $46.3 \pm 5.8$ & $<0.001$ \\
\hline PLR & $201.0 \pm 84.9$ & $183.0 \pm 95.1$ & 0.432 \\
\hline
\end{tabular}

ASA: American Society of Anesthesiologists, BMI: body mass index, IPMN: intraductal papillary mucinous neoplasm, AIP: autoimmune pancreatitis, CRP: C-reactive protein, CT: computed tomography, MRCP: magnetic resonance cholangiopancreatography, MPD: main pancreatic duct, NET: neuroendocrine tumor, SCN: Serous cystic neoplasm, PLR: platelet-to-lymphocyte ratio, PNI: prognostic nutritional index 
Values are presented as mean \pm standard deviation with 95\% confidence interval or number of patients.

\section{Operative outcomes}

No significant differences in intraoperative factors (surgical procedures, blood loss, operative time, and transfusion) were noted between the groups (Table 2). Portal vein resection was performed in two patients (10\%), both of whom had pancreatic cancer.

Table 2 Intraoperative and postoperative outcomes of patients undergoing pancreaticoduodenectomy by age group

\begin{tabular}{|c|c|c|c|}
\hline & $\begin{array}{c}\text { Elderly group } \\
(n=20)\end{array}$ & $\begin{array}{c}\text { Non-elderly group } \\
(n=102)\end{array}$ & $\begin{array}{c}P- \\
\text { value }\end{array}$ \\
\hline \multicolumn{4}{|l|}{ Intraoperative outcome } \\
\hline $\begin{array}{l}\text { Pancreaticojejunostomy technique } \\
\text { (Kakita/Blumgart) }\end{array}$ & 7/13 & $40 / 62$ & 0.722 \\
\hline Portal vein resection & $2(10)$ & $16(15.7)$ & 0.494 \\
\hline Operative time (min) & $472 \pm 82$ & $487 \pm 112$ & 0.576 \\
\hline Blood loss (mL) & $724 \pm 146$ & $643 \pm 91$ & 0.628 \\
\hline Transfusion & $5(25)$ & $21(20.1)$ & 0.665 \\
\hline Microscopic positive margin & $8(40)$ & $13(16.3)$ & 0.027 \\
\hline \multicolumn{4}{|l|}{ Postoperative outcomes } \\
\hline POPF & & & 0.854 \\
\hline Grade A & $3(15)$ & $24(23.5)$ & \\
\hline Grade B & $6(30)$ & $27(26.5)$ & \\
\hline Grade C & $1(5)$ & $4(3.9)$ & \\
\hline $\mathrm{POPF} \geq \mathrm{B}$ & 7 (35) & $31(30.4)$ & 0.688 \\
\hline Clavien-Dindo classification & & & 0.948 \\
\hline 0 & $5(25)$ & $33(32.3)$ & \\
\hline I & $3(15)$ & $17(16.7)$ & \\
\hline II & $1(5)$ & $8(7.8)$ & \\
\hline IIIa or IIIb & $10(50)$ & $41(40.1)$ & \\
\hline IV & $1(5)$ & $3(2.9)$ & \\
\hline Clavien-Dindo classification $\geq$ III & $11(55)$ & $44(43.1)$ & 0.331 \\
\hline UICC stage & & & 0.554 \\
\hline 0 & $0(0)$ & $1(1.3)$ & \\
\hline I & $4(20)$ & $23(28.8)$ & \\
\hline II & $13(65)$ & $44(55)$ & \\
\hline III & $1(5)$ & $8(10)$ & \\
\hline IV & $2(10)$ & $4(5)$ & \\
\hline Duration of in-hospital stay (days) & $39.9 \pm 28.5$ & $27 \pm 14.8$ & 0.004 \\
\hline Postoperative chemotherapy & 7 (35) & $51(50)$ & 0.216 \\
\hline $\begin{array}{l}\text { Postoperative adjuvant chemotherapy in patients with } \\
\text { pancreatic cancer }\end{array}$ & $4(57)$ & $36(80)$ & 0.182 \\
\hline Perioperative mortality & $1(5)$ & $3(2.9)$ & 0.636 \\
\hline $\begin{array}{l}\text { Cause of death } \\
\text { Cancer specific/other }\end{array}$ & $9 / 6$ & $32 / 14$ & 0.498 \\
\hline
\end{tabular}

POPF: postoperative pancreatic fistula, UICC: Union for International Cancer Control

Values are presented as mean \pm standard deviation with $95 \%$ confidence interval or number of patients.

\section{Postoperative outcome}

Postoperative complications are presented in Table 2. There was no significant difference in complications and major complications (Clavien-Dindo classification $\geq \mathrm{III}$ ) between the groups. The incidence of postoperative pancreatic fistula (POPF) was similar between the groups. The duration of in- 
hospital stay was significantly longer in the elderly group than in the non-elderly group $(P=0.004)$. UICC staging distribution was not significantly different between the groups $(P=0.554)$. One patient $(5 \%)$ died within 90 days after surgery in the elderly group. There was no significant difference in the mortality rate between the groups $(P=0.636)$. The rate of postoperative adjuvant chemotherapy for pancreatic cancer was lower in the elderly group than in the non-elderly group, although this was not significant (4 [57.1\%] vs. 36 [80.0\%]; $P=0.208)$.

\section{Change in nutritional indexes}

The preoperative albumin level and PNI were significantly lower in the elderly group than in the nonelderly group (Table 2). No differences in the preoperative hemoglobin level and platelet count were observed between the groups. The change in nutritional indexes examined before and after surgery is shown in Fig. 1a-f. Compared to the non-elderly group, the elderly group showed significant decreases in the lymphocyte count before surgery; albumin level before surgery and at 1 week, 1 month, 3 months, 6 months, and 1 year postoperatively; and PNI before surgery and at 3 months postoperatively. Those results may indicate that elderly patients had little protein reserves and impaired immune defenses compared to non-elderly patients.

\section{Long-term survival}

The median overall survival was 17.5 months in the elderly group and 45.7 months in the non-elderly group. Additionally, the 1-, 3-, and 5-year survival rates were $78.1 \%, 26.7 \%$, and $13.3 \%$, respectively, in the elderly group and $87.3 \%, 54.4 \%$, and $46.7 \%$, respectively, in the non-elderly group. Elderly patients had a significantly lower overall survival rate than non-elderly patients (log-rank test, $P=0.008$ ) (Fig. 2). When the population was categorized according to malignant tumors, including pancreatic cancer, bile duct cancer, ampullary cancer, and gallbladder cancer, survival did not significantly differ between the groups $(P=0.099)$ (Fig. 3). The median overall survival times were 17.5 months for the elderly patients with malignant tumors and 30.0 months for the non-elderly patients with malignant tumors.

\section{Causes of death}

We also analyzed the causes of death. Of 20 elderly patients, nine died of disease recurrence, whereas six died of other causes. Of 102 non-elderly patients, 32 died of disease recurrence, whereas 14 died of other causes. The rate of death due to other causes was higher in the elderly group than in the non-elderly group, although this was not significant (6 [30.0\%] vs. 14 [13.7\%]; $P=0.072$ ). Other causes of death included pneumonia in six patients; myocardial infarction in three patients; sepsis from abdominal abscess in three patients; cholangitis in two patients; malnutrition in two patients; other cancer in one patient; and unknown in two patients.

\section{Discussion}


There have been an increasing number of publications reporting on the outcomes of PD in patients aged 80 years and older instead of younger patients [3, 8, 19-22]. A systematic review and meta-analysis indicated that compared to younger patients, elderly patients had a two-fold increased mortality rate [23]. The cause of death was more related to patients' comorbidities or non-specific surgical complications than other causes [9]. In our study, elderly patients had significantly more cardiac disease than nonelderly patients. The cause of death was more related to patients' comorbidities or non-specific surgical complications in elderly patients than in non-elderly patients, although this was not significant. This finding suggests that poor functional status, such as one's nutritional status and medical comorbidities, rather than age alone contributes to the increased postoperative mortality in elderly patients.

The morbidity associated with PD that is related to specific and non-specific surgical complications remains high. A systematic review showed that patients aged 80 years or older were 1.5 fold more likely to have a complication than their younger counterparts [23]. However, with regard to complications including POPF and operative factors, we found no differences between the groups, which is in line with findings reported in other published studies $[21,24,25]$. Our experience shows that PD can be performed safely in elderly patients.

The long-term survival between patients aged $>80$ years and younger patients was noted in several previous studies. There appears to be a major variation in survival after PD in these studies, which may be related to the inclusion of different pathologies in the analysis $[6,26]$. Other studies have reported a higher mortality associated with PD in patients aged 80 years and older, which often reflects the higher ASA status [9, 27]. In our study, overall survival was significantly decreased in elderly patients, as compared to that in non-elderly patients. Additionally, overall survival was decreased in elderly patients treated for a malignant tumor, although this was not significant, because more elderly patients underwent R1 resection than non-elderly patients, and had lower induction of postoperative adjuvant chemotherapy by delay recovery after PD than non-elderly patients, though not significant. A reduction in survival among elderly patients may be related to the age-related predicted lower life expectancy of those aged 80 years and older. The average life expectancy is increasing worldwide, with men and women aged 80 years and older being expected to live for another 10 years; furthermore, the life expectancy of people aged 65 years is double that of those aged 80 years and older.

In several studies, the length of hospital stay was not different between the elderly and younger groups $[24,27,28]$. It should be noted that elderly patients were far more likely than younger patients to be discharged to a rehabilitation facility instead of home [29, 30]. In this study, the length of hospital stay was significantly longer in the elderly group than in the non-elderly group; nonetheless, the postoperative complication rate was similar between the two groups. This finding may be reflective of fewer rehabilitation facilities in our rural region than in an urban region.

The nutritional assessment of our study showed that there was a significant decrease in the albumin level, PNI, and delay of recovery after PD in elderly patients compared to non-elderly patients. At 3 months after PD, PNI was significantly lower in the elderly group than in the non-elderly group. However, Miyazaki 
et al. reported that with regard to the change in PNI during the postoperative period, there was no difference between elderly patients ( $>70$ years) and younger patients, although the definition of elderly patients in their study was different from that in our study [31]. Yamashita et al. reported that there was no significant difference in changes in the serum albumin level at 3 months after PD between the elderly patients (>75 years) and younger patients, but the recovery of the serum prealbumin level from 1 to 3 months after PD in patients aged $>75$ years was significantly delayed compared to that in younger patients [32]. Furthermore, we assessed the change in nutritional status in the long term. At 1 year after $P D$, the albumin level was significantly lower in elderly patients than in non-elderly patients. Elderly patients carry the risk for a prolonged recovery or malnutrition. Nevertheless, there was no difference between the groups with respect to other nutritional and immune markers in elderly patients; thus, further analysis is necessary.

Elderly patients have a lower physiologic reserve than younger patients do [33]. Moreover, this decrease is more likely to be related to the higher incidence of comorbidities in elderly patients than in younger patients. Recently, the enhanced recovery after surgery (ERAS) program has been reported to be safe and contributed to decreasing total complication rates and length of hospital stay in the hepatobiliary field $[34,35]$. The underlying principle of the ERAS program is a multimodal perioperative protocol to attenuate the inflammatory response and potentiate patient rehabilitation after surgery [36,37]. The adaptation of the ERAS program may be effective in elderly patients to prevent decline in nutritional status and activities of daily living after surgery.

Considering the poor survival benefit of elderly patients, it is essential to carefully select candidates for PD among elderly patients based on strict indications. Our results may be in part explained by a selection bias: we excluded patients with a very high surgical risk (ASA 4), especially those with cardiovascular or pulmonary disease, from PD. However, there are currently no clear guidelines indicating the appropriate selection of elderly patients for PD.

The present study has some limitations attributable to its small sample size and the inherent nature of retrospective analysis. Prospective cohort studies conducted at multiple institutions should be performed to confirm our study results.

\section{Conclusions}

Although age seems to be associated with an increase in mortality, it does not appear to be prohibitive and somewhat reflects a higher grade of pre-existing comorbidities and low nutritional status. Careful selection of elderly patients for PD based on their preoperative status, including their nutritional status, should guide surgical decision making for this patient population.

\section{List Of Abbreviations}


PD, pancreaticoduodenectomy; PNI, prognostic nutritional index; BMI, body mass index; MPD, main pancreatic duct; UICC, Union for International Cancer Control; ASA, American Society of Anesthesiologists; POPF, postoperative pancreatic fistula; ERAS, enhanced recovery after surgery

\section{Declarations}

\section{Ethics approval and consent to participate}

The ethics committee of the Iwakuni Clinical Center approved the study protocol (approval number: 0138). Patients were not required to provide informed consent for the study because the analysis used anonymous data that were obtained after patients agreed to treatment by written consent.

\section{Consent for publication}

Not applicable.

\section{Availability of data and materials}

Not applicable.

\section{Competing interests}

The authors declare that they have no competing interests.

\section{Funding}

No funding was received for this study.

\section{Authors' contributions}

MU, HA, SN, SN, YU, HK, YK, FT, TA, KK, and KT designed the study. HA, MU, and YK treated and observed the patients. MU prepared the manuscript and performed the literature search. HA corrected and revised the manuscript. All authors read and approved the final version of the manuscript for publication.

\section{References}

1. Ballarin R, Spaggiari M, Di Benedetto F, Montalti R, Masetti M, De Ruvo N, et al. Do not deny pancreatic resection to elderly patients. J Gastrointest Surg. 2009;13:341-8.

2. Crippa S, Dominguez I, Rodriguez JR, Razo O, Thayer SP, Ryan DP, et al. Quality of life in pancreatic cancer: analysis by stage and treatment. J Gastrointest Surg. 2008;12:783 - 93; discussion 793 84..

3. Lee MK, Dinorcia J, Reavey PL, Holden MM, Genkinger JM, Lee JA, et al. Pancreaticoduodenectomy can be performed safely in patients aged 80 years and older. J Gastrointest Surg. 2010;14:1838-46. 
4. de la Fuente SG, Bennett KM, Pappas TN, Scarborough JE. Pre- and intraoperative variables affecting early outcomes in elderly patients undergoing pancreaticoduodenectomy. HPB (Oxford). 2011;13:887-92.

5. Hardacre JM, Simo K, McGee MF, Stellato TA, Schulak JA. Pancreatic resection in octogenarians. J Surg Res. 2009;156:129-32.

6. Hatzaras I, Schmidt C, Klemanski D, Muscarella P, Melvin WS, Ellison EC, et al. Pancreatic resection in the octogenarian: a safe option for pancreatic malignancy. J Am Coll Surg. 2011;212:373-7.

7. Khan S, Sclabas G, Lombardo KR, Sarr MG, Nagorney D, Kendrick ML, et al. Pancreatoduodenectomy for ductal adenocarcinoma in the very elderly; is it safe and justified? J Gastrointest Surg. 2010;14:1826-31.

8. Langan RC, Zheng C, Harris K, Verstraete R, Al-Refaie WB, Johnson LB. Hospital-level resource use by the oldest-old for pancreaticoduodenectomy at high-volume hospitals. Surgery. 2015;158:366-72.

9. Belyaev O, Herzog T, Kaya G, Chromik AM, Meurer K, Uhl W, et al. Pancreatic surgery in the very old: face to face with a challenge of the near future. World J Surg. 2013;37:1013-20.

10. Zhu XH, Wu YF, Qiu YD, Jiang CP, Ding YT. Effect of early enteral combined with parenteral nutrition in patients undergoing pancreaticoduodenectomy. World J Gastroenterol. 2013;19:5889-96.

11. Fujiwara $Y$, Shiba H, Shirai $Y$, Iwase R, Haruki K, Furukawa K, et al. Perioperative serum albumin correlates with postoperative pancreatic fistula after pancreaticoduodenectomy. Anticancer Res. 2015;35:499-503.

12. Watanabe J, Otani S, Sakamoto T, Arai Y, Hanaki T, Amisaki M, et al. Prognostic indicators based on inflammatory and nutritional factors after pancreaticoduodenectomy for pancreatic cancer. Surg Today. 2016;46:1258-67.

13. Onodera T, Goseki N, Kosaki G. Prognostic nutritional index in gastrointestinal surgery of malnourished cancer patients. Nihon Geka Gakkai Zasshi. 1984;85:1001-5.

14. Ohtsuka M, Miyakawa S, Nagino M, Takada T, Miyazaki M. Revision concepts and distinctive points of the new Japanese classification for biliary tract cancers in comparison with the 7(th) edition of the Union for International Cancer Control and the American Joint Committee on Cancer staging system. J Hepatobiliary Pancreat Sci. 2015;22:197-201.

15. Bassi C, Dervenis C, Butturini G, Fingerhut A, Yeo C, Izbicki J, et al. Postoperative pancreatic fistula: an international study group (ISGPF) definition. Surgery. 2005;138:8-13.

16. Pratt WB, Callery MP, Vollmer CM Jr. The latent presentation of pancreatic fistulas. Br J Surg. 2009;96:641-9.

17. Kouba R. Intestine plication. Opinion on the contribution of H. Merguet, Technical modification of the mesenterial plication by the Noble-Child method in "Der Chirurg" 44,308 - 10 (1973). Chirurg. 1974;45:272-3.

18. Kawakatsu S, Inoue Y, Mise Y, Ishizawa T, Ito H, Takahashi Y, et al. Comparison of pancreatojejunostomy techniques in patients with a soft pancreas: Kakita anastomosis and Blumgart anastomosis. BMC Surg. 2018;18:88. 
19. Bu XM, Xu J, Dai XW, Ma K, Yang FQ, Hu J, et al. Is delayed gastric emptying so terrible after pyloruspreserving pancreaticoduodenectomy? Prevention and management. World J Gastroenterol. 2006;12:6382-5.

20. Beltrame V, Gruppo M, Pastorelli D, Pedrazzoli S, Merigliano S, Sperti C. Outcome of pancreaticoduodenectomy in octogenarians: Single institution's experience and review of the literature. J Visc Surg. 2015;152:279-84.

21. Oguro S, Shimada K, Kishi Y, Nara S, Esaki M, Kosuge T. Perioperative and long-term outcomes after pancreaticoduodenectomy in elderly patients 80 years of age and older. Langenbecks Arch Surg. 2013;398:531-8.

22. Lee DY, Schwartz JA, Wexelman B, Kirchoff D, Yang KC, Attiyeh F. Outcomes of pancreaticoduodenectomy for pancreatic malignancy in octogenarians: an American College of Surgeons National Surgical Quality Improvement Program analysis. Am J Surg. 2014;207:540-8.

23. Kim SY, Weinberg L, Christophi C, Nikfarjam M. The outcomes of pancreaticoduodenectomy in patients aged 80 or older: a systematic review and meta-analysis. HPB (Oxford). 2017;19:475-82.

24. Kim SY, Fink MA, Perini M, Houli N, Weinberg L, Muralidharan V, et al. Age 80 years and over is not associated with increased morbidity and mortality following pancreaticoduodenectomy. ANZ J Surg. 2018;88:E445-50.

25. Tani M, Kawai M, Hirono S, Ina S, Miyazawa M, Nishioka R, et al. A pancreaticoduodenectomy is acceptable for periampullary tumors in the elderly, even in patients over 80 years of age. $\mathrm{J}$ Hepatobiliary Pancreat Surg. 2009;16:675-80.

26. Melis M, Marcon F, Masi A, Pinna A, Sarpel U, Miller G, et al. The safety of a pancreaticoduodenectomy in patients older than 80 years: risk vs. benefits. HPB (Oxford). 2012;14:583-8.

27. Makary MA, Winter JM, Cameron JL, Campbell KA, Chang D, Cunningham SC, et al. Pancreaticoduodenectomy in the very elderly. J Gastrointest Surg. 2006;10:347-56.

28. Cameron JL, He J. Two thousand consecutive pancreaticoduodenectomies. J Am Coll Surg. 2015;220:530-6.

29. Dale W, Hemmerich J, Kamm A, Posner MC, Matthews JB, Rothman R, et al. Geriatric assessment improves prediction of surgical outcomes in older adults undergoing pancreaticoduodenectomy: a prospective cohort study. Ann Surg. 2014;259:960-5.

30. Riall TS, Reddy DM, Nealon WH, Goodwin JS. The effect of age on short-term outcomes after pancreatic resection: a population-based study. Ann Surg. 2008;248:459-67.

31. Miyazaki Y, Kokudo T, Amikura K, Kageyama Y, Takahashi A, Ohkohchi N, et al. Age does not affect complications and overall survival rate after pancreaticoduodenectomy: single-center experience and systematic review of literature. Biosci Trends. 2016;10:300-6.

32. Yamashita Y, Shirabe K, Tsujita E, Takeishi K, Ikeda T, Yoshizumi T, et al. Surgical outcomes of pancreaticoduodenectomy for periampullary tumors in elderly patients. Langenbecks Arch Surg. 2013;398:539-45. 
33. Tzeng CW, Cooper AB, Vauthey JN, Curley SA, Aloia TA. Predictors of morbidity and mortality after hepatectomy in elderly patients: analysis of 7621 NSQIP patients. HPB (Oxford). 2014;16:459-68.

34. Ji HB, Zhu WT, Wei Q, Wang XX, Wang HB, Chen QP. Impact of enhanced recovery after surgery programs on pancreatic surgery: a meta-analysis. World J Gastroenterol. 2018;24:1666-78.

35. Hughes MJ, McNally S, Wigmore SJ. Enhanced recovery following liver surgery: a systematic review and meta-analysis. HPB (Oxford). 2014;16:699-706.

36. Kehlet $\mathrm{H}$. Multimodal approach to control postoperative pathophysiology and rehabilitation. $\mathrm{Br} \mathrm{J}$ Anaesth. 1997;78:606-17.

37. Kehlet $\mathrm{H}$, Wilmore DW. Evidence-based surgical care and the evolution of fast-track surgery. Ann Surg. 2008;248:189-98.

\section{Figures}

a

8
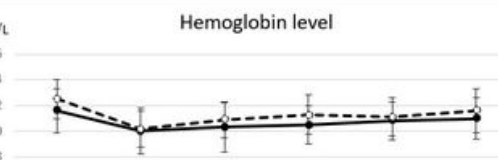

before 1 week 1 month 3 months 6 months 1 year

$\rightarrow 280$ years $\quad-\infty-<80$ years

b

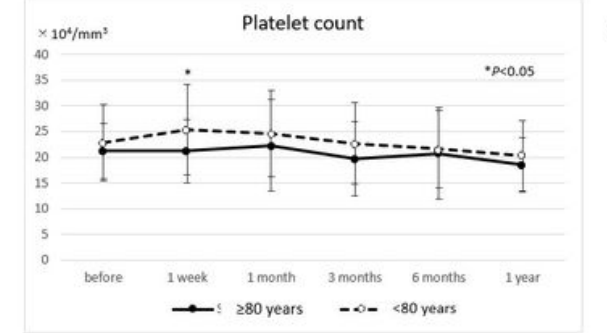

c

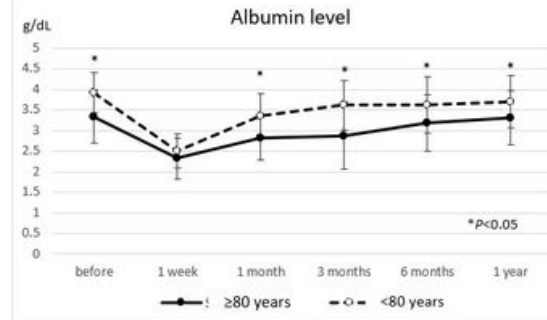

d

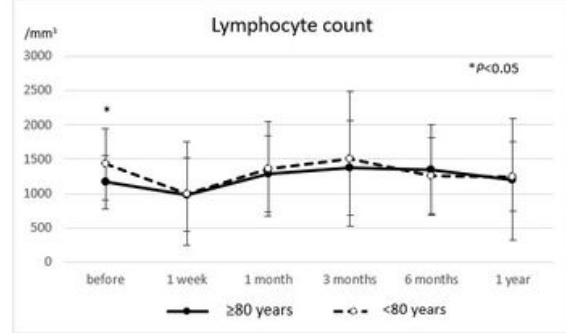

e

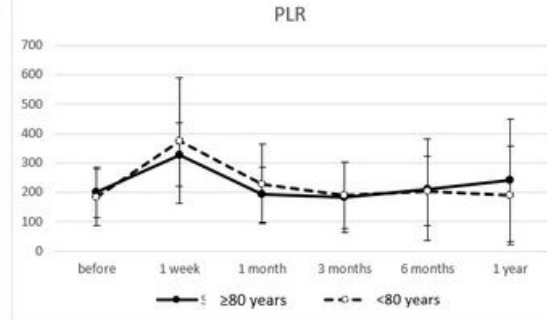

i

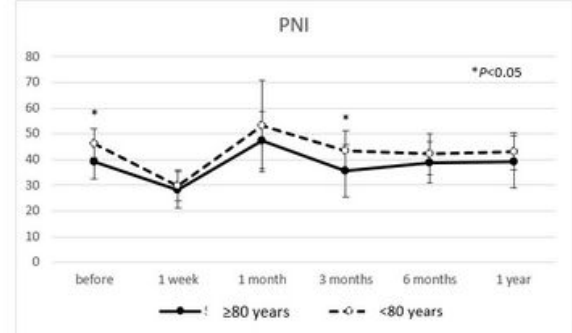

\section{Figure 1}

Change in nutritional indexes before and after surgery. a Hemoglobin level, b platelet count, c albumin level, d lymphocyte count, e platelet-to-lymphocyte ratio (PLR), f prognostic nutritional index (PNI) 


\section{All patients}

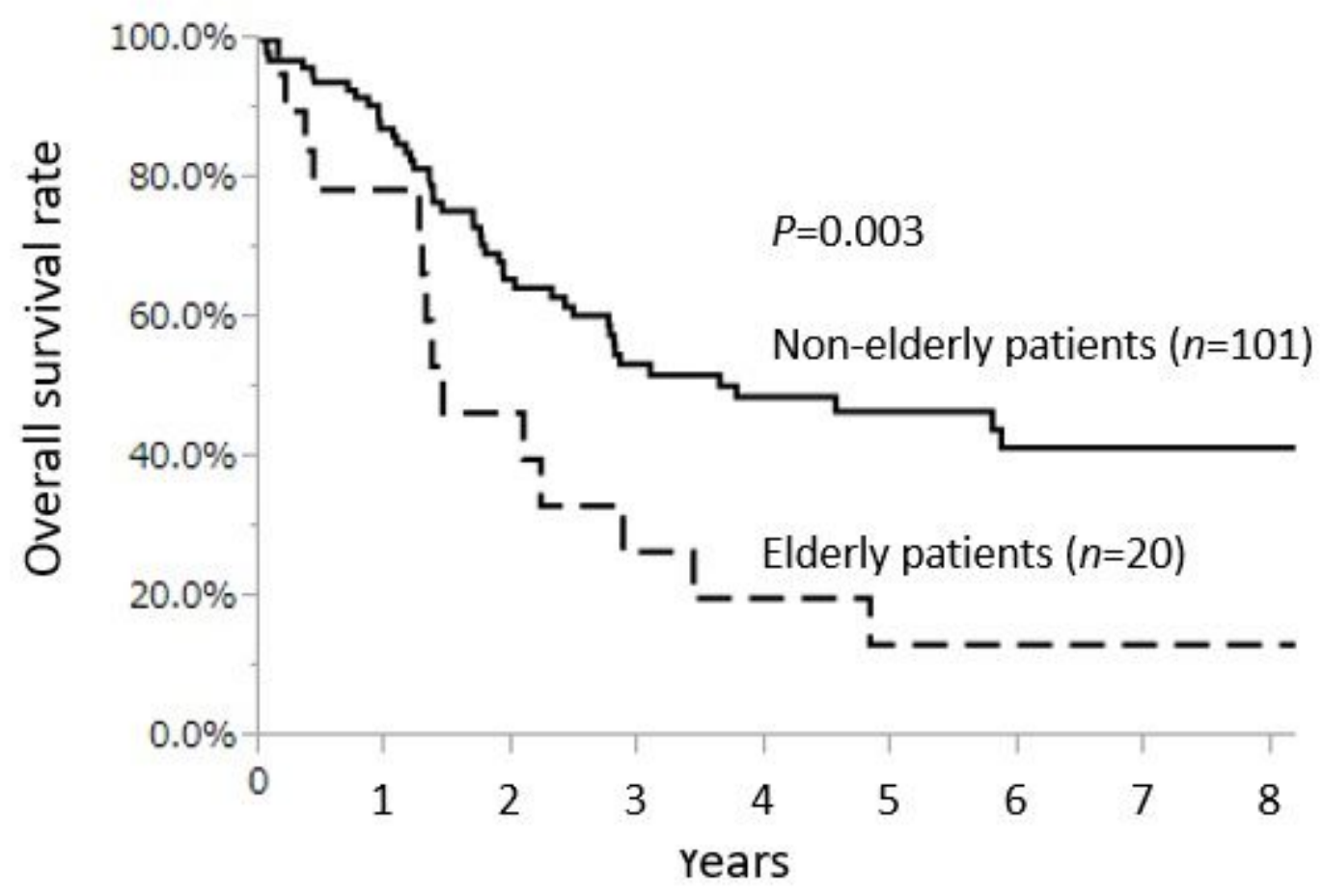

Figure 2

Overall survival curves for all the study population comparing elderly patients undergoing pancreaticoduodenectomy with the non-elderly patients. The median overall survival was 17.5 months in the elderly group and 45.7 months in the non-elderly group. Additionally, the 1-, 3-, and 5-year survival rates were $78.1 \%, 26.7 \%$, and $13.3 \%$, respectively, in the elderly group and $87.3 \%, 54.4 \%$, and $46.7 \%$, respectively, in the non-elderly group. Elderly patients had a significantly lower overall survival rate than non-elderly patients (log-rank test, $\mathrm{P}=0.008$ ) 


\section{Malignant tumor}

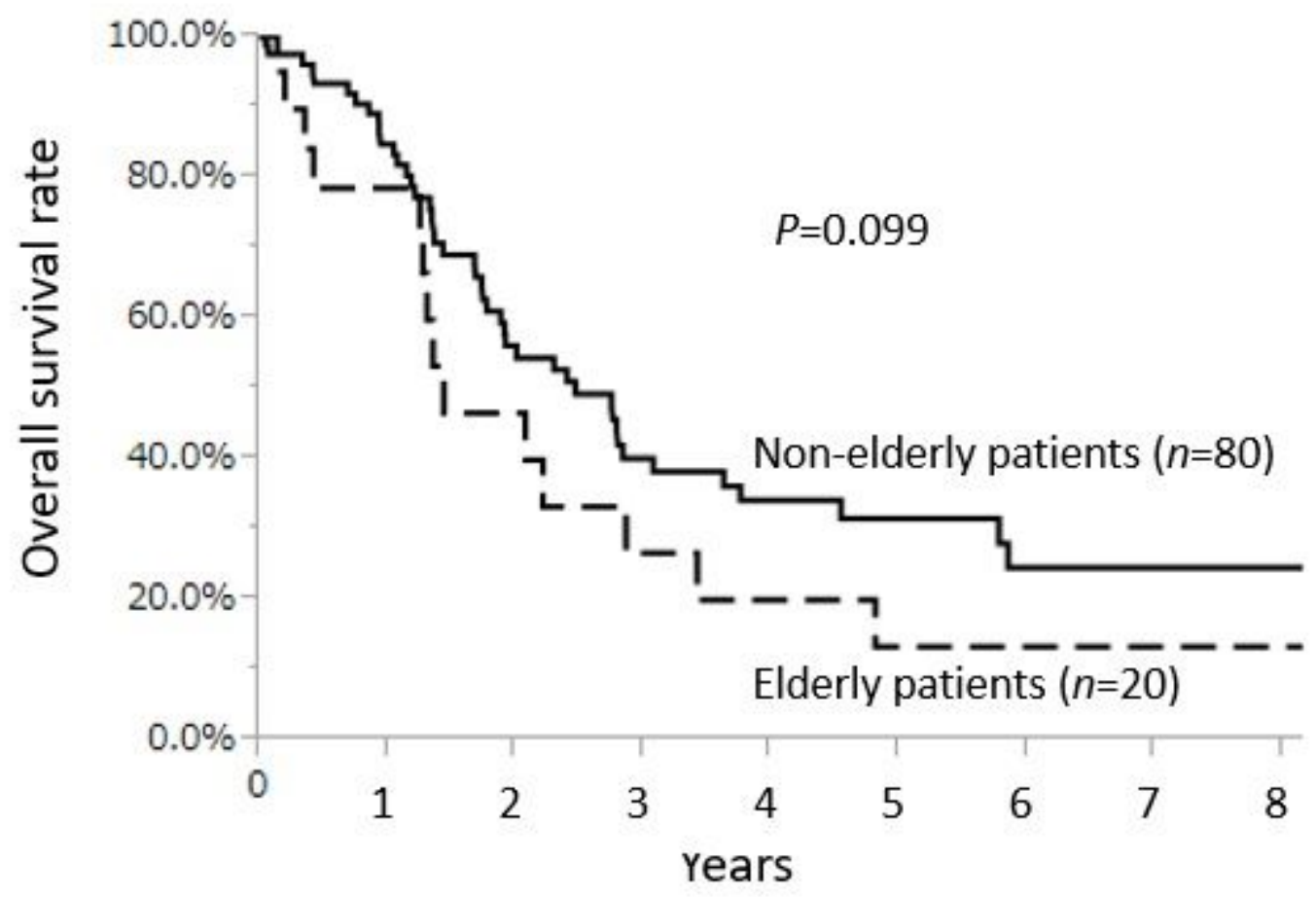

Figure 3

Overall survival curves comparing elderly patients undergoing pancreaticoduodenectomy for malignant tumors with the non-elderly group. Overall survival did not significantly differ between the groups $(P=0.099)$. The median overall survival times were 17.5 months for the elderly patients with malignant tumors and 30.0 months for the non-elderly patients with malignant tumors. 